\title{
ON WEAKLY GROUP-THEORETICAL NON-DEGENERATE BRAIDED FUSION CATEGORIES
}

\author{
SONIA NATALE
}

\begin{abstract}
We show that the Witt class of a weakly group-theoretical non-degenerate braided fusion category belongs to the subgroup generated by classes of non-degenerate pointed braided fusion categories and Ising braided categories. This applies in particular to solvable nondegenerate braided fusion categories. We also give some sufficient conditions for a braided fusion category to be weakly group-theoretical or solvable in terms of the factorization of its Frobenius-Perron dimension and the Frobenius-Perron dimensions of its simple objects. As an application, we prove that every non-degenerate braided fusion category whose Frobenius-Perron dimension is a natural number less than 1800, or an odd natural number less than 33075 , is weakly group-theoretical.
\end{abstract}

\section{INTRODUCTION}

Let $k$ be an algebraically closed field of characteristic zero. A fusion category over $k$ is a finite semisimple rigid tensor category $\mathcal{C}$ over $k$.

The notion of nilpotency of a fusion category was introduced in [[ए3], extending the corresponding one for finite groups: a fusion category is nilpotent if it can be obtained from the category Vec of finite dimensional vector spaces over $k$ by means of a finite number of finite group extensions.

A fusion category $\mathcal{C}$ is called weakly group-theoretical if it is categorically Morita equivalent to a nilpotent fusion category, that is, if there exists an indecomposable module category $\mathcal{M}$ such that the category $\mathcal{C}_{\mathcal{M}}^{*}$ of $\mathcal{C}$-module endofunctors of $\mathcal{M}$ is a nilpotent fusion category. In particular, every weakly group-theoretical fusion category has integer Frobenius-Perron dimension. If, furthermore, $\mathcal{C}$ is Morita equivalent to a cyclically nilpotent fusion category, then $\mathcal{C}$ is called solvable. Equivalently, $\mathcal{C}$ is solvable if there exist sequences Vec $=\mathcal{C}_{0}, \ldots, \mathcal{C}_{n}=\mathcal{C}$, of fusion categories, and $G_{1}, \ldots, G_{n}$, of cyclic groups of prime order such that for all $1 \leq i \leq n, \mathcal{C}_{i}$ is a $G_{i^{-}}$ equivariantization or a $G_{i}$-extension of $\mathcal{C}_{i-1}$. See Subsection 22. We refer the reader to [ $\square]$ for other characterizations and main properties of weakly group-theoretical and related fusion categories.

Date: January 22, 2014.

2010 Mathematics Subject Classification. 18D10.

Key words and phrases. Braided fusion category; braided $G$-crossed fusion category; Tannakian category; Witt class; solvability.

Partially supported by CONICET and SeCYT-UNC. 
An important class of fusion categories is that of braided fusion categories, that is, fusion categories $\mathcal{C}$ endowed with natural isomorphisms $c: X \otimes Y \rightarrow$ $Y \otimes X, X, Y \in \mathcal{C}$, called a braiding, subject to appropriate axioms. Two extreme classes of braided fusion categories, so-called symmetric and nondegenerate braided fusion categories, appear related to the square of the braiding.

Symmetric fusion categories have been classified by Deligne [ $[\mathbf{]}$. On the other side, a number of important results concerning the structure of a nondegenerate braided fusion category have been established in the literature. A non-degenerate braided fusion category endowed with a compatible ribbon structure is called a modular category. Modular categories have many applications in distinct areas of mathematics and mathematical physics, for instance, in low-dimensional topology, they constitute an important tool in the construction of invariants of knots and 3-manifolds. See e.g. [U, ए2].

The group of Witt classes of non-degenerate braided fusion categories, denoted $\mathcal{W}$, was introduced in [ [U]. Two non-degenerate braided fusion categories $\mathcal{C}_{1}$ and $\mathcal{C}_{2}$ are called Witt equivalent if there exist fusion categories $\mathcal{D}_{1}$ and $\mathcal{D}_{2}$ such that $\mathcal{C}_{1} \otimes \mathcal{Z}\left(\mathcal{D}_{1}\right) \cong \mathcal{C}_{2} \otimes \mathcal{Z}\left(\mathcal{D}_{2}\right)$ as braided tensor categories, where $\mathcal{Z}\left(\mathcal{D}_{i}\right)$ denotes the Drinfeld center of the fusion category $\mathcal{D}_{i}, i=1,2$.

The Witt group $\mathcal{W}$ consists of equivalence classes of non-degenerate braided fusion categories under this equivalence relation with multiplication induced by Deligne's tensor product $\otimes$. The unit element is the class of the category Vec of finite-dimensional vector spaces over the base field $k$ and the inverse of the class of a non-degenerate braided fusion category $\mathcal{C}$ is the class of the reverse braided fusion category $\mathcal{C}^{\text {rev }}$. This endows $\mathcal{W}$ with the structure of an (infinite countable) abelian group.

The explicit determination of the structure of the group $\mathcal{W}$ and the relations amongst its elements are pointed out in [0] as relevant problems in connection with the classification of fusion categories.

Let $\mathcal{W}_{p t}$ and $\mathcal{W}_{\text {Ising }}$ denote, respectively, the subgroup of Witt classes of pointed non-degenerate fusion categories and the subgroup generated by Witt classes of Ising braided categories.

Recall that an Ising braided category is a non-pointed braided fusion category of Frobenius-Perron dimension 4. Ising braided categories were classified in [9, Appendix B]; it is known that they fall into 8 equivalence classes and all of them are non-degenerate. If $\mathcal{I}$ is an Ising braided category, then the pointed subcategory $\mathcal{I}_{p t}$ is the unique nontrivial (symmetric) subcategory of $\mathcal{I}$, and it is equivalent to the category sVec of super-vector spaces. Besides, $\mathcal{I}$ has a unique non-invertible simple object of Frobenius-Perron dimension $\sqrt{2}$.

The subgroups $\mathcal{W}_{p t}$ and $\mathcal{W}_{\text {Ising }}$ are explicitly described in [ [Q], Appendix A.7 and Appendix B]; see also [G, Sections 5.3 and 6.4 (3)]. We have that $\mathcal{W}_{\text {Ising }}$ is isomorphic to the cyclic group of order 16 . On the other hand, if 
$\mathcal{W}_{p t}(p)$ denotes the group of classes of metric $p$-groups, we have an isomorphism $\mathcal{W}_{p t} \cong \bigoplus_{p \text { prime }} \mathcal{W}_{p t}(p)$. In addition, $\mathcal{W}_{p t}(2) \simeq \mathbb{Z}_{8} \oplus \mathbb{Z}_{2}, \mathcal{W}_{p t}(p) \simeq \mathbb{Z}_{4}$, if $p=3(\bmod 4)$, and $\mathcal{W}_{p t}(p) \simeq \mathbb{Z}_{2} \oplus \mathbb{Z}_{2}$, if $p=1(\bmod 4)$.

In this paper we show that if $\mathcal{C}$ is a non-degenerate braided fusion category such that $\mathcal{C}$ is weakly group-theoretical, then the Witt class $[\mathcal{C}]$ of $\mathcal{C}$ belongs to the subgroup generated by $\mathcal{W}_{p t}$ and $\mathcal{W}_{\text {Ising. }}$ If, moreover, $\mathcal{C}$ is integral, then $[\mathcal{C}] \in \mathcal{W}_{p t}$. See Theorem [2.2].

The proof of Theorem [.2 is given in Section [6. It relies on results of the paper [ $[$ ]. It makes use as well of the notion of a braided group-crossed fusion category introduced by Turaev [20], 24] and its main properties, in particular, its connection with the existence of nontrivial Tannakian subcategories in a braided fusion category. These results are recalled in Sections $\mathbf{3}$ and $\mathbf{0}$. Using these tools, we also prove in Section 15 a related result (Theorem 5.3 ) on the structure of solvable braided fusion categories.

Let $\widetilde{\mathcal{W}}$ be the subgroup of $\mathcal{W}$ generated by Witt equivalence classes of the fusion categories $\mathcal{C}(\mathfrak{g}, l)$ of integrable highest weight modules of level $l \in \mathbb{Z}_{+}$over the affinization of a simple finite-dimensional Lie algebra $\mathfrak{g}$. It is shown in [G] that $\mathcal{W}_{p t}, \mathcal{W}_{\text {Ising }} \subseteq \widetilde{\mathcal{W}}$. Conjecturally, $\widetilde{\mathcal{W}}$ coincides with the subgroup $\mathcal{W}_{\text {un }}$ of Witt classes of pseudo-unitary non-degenerate braided fusion categories [ [Q

On the other side, it is also conjectured that every fusion category of integer Frobenius-Perron dimension is weakly group-theoretical [प], Question 2]. As a consequence of Theorem [.2, we obtain that for every nondegenerate braided fusion category $\mathcal{C}$ such that $\mathcal{C}$ is weakly group-theoretical, then $[\mathcal{C}] \in \widetilde{\mathcal{W}}$.

One of the main results of [ $[\mathbb{U}]$ establishes the analogue of Burnside's $p^{a} q^{b}$-theorem for fusion categories, namely, that any fusion category $\mathcal{C}$ whose Frobenius-Perron dimension is $p^{a} q^{b}$, where $p$ and $q$ are prime numbers and $a, b$ are non-negative integers, is solvable. Some solvability results for braided fusion categories have been obtained in [ए], [20]. In particular, if $\mathcal{C}$ is a braided fusion category such that the Frobenius-Perron dimensions of simple objects of $\mathcal{C}$ are $\leq 2$, or if $\mathrm{FPdim} \mathcal{C}$ is odd and the Frobenius-Perron dimensions of simple objects of $\mathcal{C}$ are powers of a fixed prime number, then $\mathcal{C}$ is solvable.

Combining the main properties of braided group-crossed fusion categories with the methods developed in the paper [प], we also give in Section $\square$ some further sufficient conditions for a braided fusion category to be solvable or weakly group-theoretical. We show that every weakly integral braided fusion category whose Frobenius-Perron dimensions of simple objects are powers of a fixed prime number is always solvable. See Theorem [r.2. This extends the previously mentioned results in [प], [U]].

In addition, we show that every non-degenerate braided fusion category $\mathcal{C}$ whose Frobenius-Perron dimension factorizes in the form $\operatorname{FPdim} \mathcal{C}=p^{a} q^{b} c$, 
where $p$ and $q$ are prime numbers, $a$ and $b$ are nonnegative integers, and $c$ is a square-free natural number, is necessarily weakly group-theoretical. See Theorem [.].

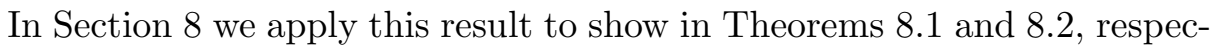
tively, that every weakly integral non-degenerate braided fusion category of Frobenius-Perron dimension less than 1800 is weakly group-theoretical and moreover, it is solvable if its Frobenius-Perron dimension is odd and less than 33075 .

Acknowledgement. This paper was written during a visit to the Institute des Hautes Etudes Scientifiques, France. The author is grateful to the IHES for the outstanding hospitality and the excellent atmosphere.

\section{Preliminaries AND NOTATiON}

We shall work over an algebraically closed field $k$ of characteristic zero. The category of finite dimensional vector spaces over $k$ will be denoted by Vec. A fusion category over $k$ is a semisimple rigid monoidal category over $k$ with finitely many isomorphism classes of simple objects, finite-dimensional Hom spaces, and such that the unit object $\mathbf{1}$ is simple. We refer the reader to $[\mathbb{U}, \mathbb{\square}, \mathbb{Q}]$ for the main notions about fusion categories and braided fusion categories used throughout. Unless otherwise stated, all tensor categories will be assumed to be strict.

2.1. Frobenius-Perron dimensions. Let $\mathcal{C}$ be a fusion category. The Frobenius-Perron dimension of a simple object $X \in \mathcal{C}$ is, by definition, the Frobenius-Perron eigenvalue of the matrix of left multiplication by the class of $X$ in the basis $\operatorname{Irr}(\mathcal{C})$ of the Grothendieck ring of $\mathcal{C}$ consisting of isomorphism classes of simple objects. The Frobenius-Perron dimension of $\mathcal{C}$ is the number $\mathrm{FPdim} \mathcal{C}=\sum_{X \in \operatorname{Irr}(\mathcal{C})}(\mathrm{FP} \operatorname{dim} X)^{2}$. The category $\mathcal{C}$ is called integral if $\operatorname{FPdim} X \in \mathbb{Z}$, for all simple object $X \in \mathcal{C}$, and it is called weakly integral if $\mathrm{FPdim} \mathcal{C} \in \mathbb{Z}$.

If $\mathcal{C}$ is a weakly integral fusion category, then $(\mathrm{FPdim} X)^{2} \in \mathbb{Z}$, for all simple object $X \in \mathcal{C}$ [س], Proposition 8.27]. This implies, in particular, that a fusion subcategory of $\mathcal{C}$ is also weakly integral. On the other hand, if $\mathcal{C}$ is weakly integral (respectively, integral) and $F: \mathcal{C} \rightarrow \mathcal{D}$ is a dominant (or surjective) tensor functor, then $\mathcal{D}$ is weakly integral (respectively, integral) as well; see [, Proposition 2.12], [面, Corollary 8.36].

2.2. Nilpotent and weakly group-theoretical fusion categories. Let $G$ be a finite group. A $G$-grading on a fusion category $\mathcal{C}$ is a decomposition $\mathcal{C}=\oplus_{g \in G} \mathcal{C}_{g}$, such that $\mathcal{C}_{g} \otimes \mathcal{C}_{h} \subseteq \mathcal{C}_{g h}$ and $\mathcal{C}_{g}^{*} \subseteq \mathcal{C}_{g^{-1}}$, for all $g, h \in G$. The fusion category $\mathcal{C}$ is called a $G$-extension of a fusion category $\mathcal{D}$ if there is a faithful grading $\mathcal{C}=\oplus_{g \in G} \mathcal{C}_{g}$ with neutral component $\mathcal{C}_{e} \cong \mathcal{D}$.

If $\mathcal{C}$ is any fusion category, there exist a finite group $U(\mathcal{C})$, called the universal grading group of $\mathcal{C}$, and a canonical faithful grading $\mathcal{C}=\oplus_{g \in U(\mathcal{C})} \mathcal{C}_{g}$, 
with neutral component $\mathcal{C}_{e}=\mathcal{C}_{a d}$, where $\mathcal{C}_{a d}$ is the adjoint subcategory of $\mathcal{C}$, that is, the fusion subcategory generated by $X \otimes X^{*}, X \in \operatorname{Irr}(\mathcal{C})$.

A fusion category $\mathcal{C}$ is (cyclically) nilpotent if there exists a sequence of fusion categories Vec $=\mathcal{C}_{0} \subseteq \mathcal{C}_{1} \cdots \subseteq \mathcal{C}_{n}=\mathcal{C}$, and finite (cyclic) groups $G_{1}, \ldots, G_{n}$, such that for all $i=1, \ldots, n, \mathcal{C}_{i}$ is a $G_{i}$-extension of $\mathcal{C}_{i-1}$.

Dual to the notion of a group extension, we have the notion of an equivariantization. Consider an action of a finite group $G$ on a fusion category $\mathcal{C}$ by tensor autoequivalences $\rho: \underline{G} \rightarrow \underline{\mathrm{Aut}}_{\otimes} \mathcal{C}$. The equivariantization of $\mathcal{C}$ with respect to the action $\rho$, denoted $\mathcal{C}^{G}$, is a fusion category whose objects are pairs $(X, \mu)$, such that $X$ is an object of $\mathcal{C}$ and $\mu=\left(\mu^{g}\right)_{g \in G}$, is a collection of isomorphisms $\mu^{g}: \rho^{g} X \rightarrow X, g \in G$, satisfying appropriate compatibility conditions.

The forgetful functor $F: \mathcal{C}^{G} \rightarrow \mathcal{C}, F(X, \mu)=X$, is a dominant tensor functor that gives rise to a central exact sequence of fusion categories $\operatorname{Rep} G \rightarrow \mathcal{C}^{G} \rightarrow \mathcal{C}[\mathbb{Z}]$, where $\operatorname{Rep} G$ is the category of finite-dimensional representations of $G$.

A module category over a fusion category $\mathcal{C}$ is a finite semisimple $k$-linear abelian category $\mathcal{M}$ endowed with a bifunctor $\otimes: \mathcal{C} \times \mathcal{M} \rightarrow \mathcal{M}$ satisfying the associativity and unit axioms for an action, up to coherent natural isomorphisms.

The module category $\mathcal{M}$ is called decomposable if it is equivalent as a module category to a direct sum of non-trivial module categories, and it is called indecomposable otherwise. If $\mathcal{M}$ is an indecomposable module category over $\mathcal{C}$, then the category $\mathcal{C}_{\mathcal{M}}^{*}$ of $\mathcal{C}$-module endofunctors of $\mathcal{M}$ is also a fusion category.

Two fusion categories $\mathcal{C}$ and $\mathcal{D}$ are Morita equivalent if $\mathcal{D}$ is equivalent to $\mathcal{C}_{\mathcal{M}}^{*}$ for some indecomposable module category $\mathcal{M}$.

A fusion category $\mathcal{C}$ is called group-theoretical if it is Morita equivalent to a pointed fusion category, that is, to a fusion category all of whose simple objects are invertible. On the other hand, $\mathcal{C}$ is called weakly group-theoretical (respectively, solvable) if it is Morita equivalent to a nilpotent (respectively, cyclically nilpotent) fusion category.

It is shown in [ய, Proposition 4.1] that the class of weakly group-theoretical fusion categories is stable under the operations of taking extensions, equivariantizations, Morita equivalent categories, tensor products, Drinfeld center, fusion subcategories and components of quotient categories. Also, the class of solvable fusion categories is stable under taking extensions and equivariantizations by solvable groups, Morita equivalent categories, tensor products, Drinfeld center, fusion subcategories and components of quotient categories.

2.3. Braided fusion categories. A braiding in a fusion category $\mathcal{C}$ is a natural isomorphism $c_{X, Y}: X \otimes Y \rightarrow Y \otimes X, X, Y \in \mathcal{C}$, subject to the 
hexagon axioms. A braided fusion category is a fusion category endowed with a braiding.

Suppose $\mathcal{C}$ is a braided fusion category. The reverse braided fusion category will be denoted by $\mathcal{C}^{\text {rev}}$; thus, if $c_{X, Y}: X \otimes Y \rightarrow Y \otimes X$ denotes the braiding of $\mathcal{C}$, then $\mathcal{C}^{\text {rev }}=\mathcal{C}$ as a fusion category, with braiding $c_{X, Y}^{\text {rev }}=c_{Y, X}^{-1}$, for all objects $X, Y$.

If $\mathcal{D}$ is a fusion subcategory of a braided fusion category $\mathcal{C}$, the Müger centralizer of $\mathcal{D}$ in $\mathcal{C}$ will be denoted by $\mathcal{Z}_{2}(\mathcal{D}, \mathcal{C})$, or also by $\mathcal{D}^{\prime}$ when there is no ambiguity. Thus $\mathcal{Z}_{2}(\mathcal{D}, \mathcal{C})$ is the full fusion subcategory generated by all objects $X \in \mathcal{C}$ such that $c_{Y, X} c_{X, Y}=\mathrm{id}_{X \otimes Y}$, for all objects $Y \in \mathcal{D}$.

The Müger (or symmetric) center of $\mathcal{C}$ will be denoted by $\mathcal{Z}_{2}(\mathcal{C}):=$ $\mathcal{Z}_{2}(\mathcal{C}, \mathcal{C})$. The category $\mathcal{C}$ is called symmetric if $\mathcal{Z}_{2}(\mathcal{C})=\mathcal{C}$. If $\mathcal{C}$ is any braided fusion category, its Müger center $\mathcal{Z}_{2}(\mathcal{C})$ is a symmetric fusion subcategory of $\mathcal{C}$. On the opposite extreme, $\mathcal{C}$ is called non-degenerate (respectively, slightly degenerate) if $\mathcal{Z}_{2}(\mathcal{C}) \cong \operatorname{Vec}$ (respectively, if $\mathcal{Z}_{2}(\mathcal{C}) \cong \mathrm{sVec}$ ).

For a fusion category $\mathcal{C}$, the Drinfeld center of $\mathcal{C}$ will be denoted $\mathcal{Z}(\mathcal{C})$. It is known that $\mathcal{Z}(\mathcal{C})$ is a braided non-degenerate fusion category of FrobeniusPerron dimension FPdim $\mathcal{Z}(\mathcal{C})=(\text { FPdim } \mathcal{C})^{2}$. Necessary and sufficient conditions for a braided fusion category to be equivalent to the center of some fusion category are given in [Q]].

Let $G$ be a finite group. The fusion category of finite dimensional representations of $G$ will be denoted by $\operatorname{Rep} G$. This is a symmetric fusion category with respect to the canonical braiding. A braided fusion category $\mathcal{E}$ is called Tannakian, if $\mathcal{E} \cong \operatorname{Rep} G$ for some finite group $G$ as symmetric fusion categories.

A Theorem of Deligne [ [], states that every symmetric fusion category $\mathcal{L}$ is super-Tannakian, meaning that there exist a finite group $G$ and a central element $u \in G$ of order 2 , such that $\mathcal{L}$ is equivalent to the category $\operatorname{Rep}(G, u)$ of representations of $G$ on finite-dimensional super-vector spaces where $u$ acts as the parity operator.

Hence if $\mathcal{L} \cong \operatorname{Rep}(G, u)$ is a symmetric fusion category, then $\mathcal{E}=\operatorname{Rep} G / u$ is a Tannakian subcategory of $\mathcal{L}$ and $\operatorname{FPdim} \mathcal{E}=\operatorname{FPdim} \mathcal{L} / 2$; in particular, if $\operatorname{FPdim} \mathcal{L}>2$, then $\mathcal{L}$ necessarily contains a Tannakian subcategory, and a non-Tannakian symmetric fusion category of Frobenius-Perron dimension 2 is equivalent to the category $\mathrm{sVec}$ of finite-dimensional super-vector spaces. See [ய], Section 2.4].

\section{Connected Étale Algebras in BRAided Fusion CATEgories}

Let $\mathcal{C}$ be a braided fusion category. Recall from [ $[$ ] that a separable commutative algebra $A \in \mathcal{C}$ is called an étale algebra in $\mathcal{C}$. If $\operatorname{Hom}_{\mathcal{C}}(\mathbf{1}, A) \cong$ $k$, then $A$ is called connected.

Let $A \in \mathcal{C}$ be a connected étale algebra. Let $\mathcal{C}_{A}$ denote the category of right $A$-modules in $\mathcal{C}$. Every right $A$-module $X$, with action $\rho: X \otimes A \rightarrow X$, 
is endowed with a left $A$-module structure defined by $\rho c_{X, A}^{-1}: A \otimes X \rightarrow X$, that makes it into an $A$-bimodule in $\mathcal{C}$. In this way, the category $\mathcal{C}_{A}$ becomes a fusion category with tensor product $\otimes_{A}$ and unit object $A$.

Let also $\mathcal{C}_{A}^{0}$ be the category of dyslectic $A$-modules in $\mathcal{C}$, that is, $\mathcal{C}_{A}^{0}$ is the full subcategory of $\mathcal{C}_{A}$ whose objects are right $A$-modules $X$ in $\mathcal{C}$ such that the action $\rho: X \otimes A \rightarrow X$ satisfies $\rho c_{A, X} c_{X, A}=\rho$. The category $\mathcal{C}_{A}^{0}$ is fusion subcategory of $\mathcal{C}_{A}$ and it is moreover braided with braiding induced from that of $\mathcal{C}$.

If $\mathcal{C}$ is a non-degenerate braided fusion category, then there is an equivalence of braided fusion categories

$$
\mathcal{C} \otimes\left(\mathcal{C}_{A}^{0}\right)^{\mathrm{rev}} \cong \mathcal{Z}\left(\mathcal{C}_{A}\right),
$$

such that the restriction of the forgetful functor $U: \mathcal{Z}\left(\mathcal{C}_{A}\right) \rightarrow \mathcal{C}_{A}$ to $\mathcal{C} \cong$ $\mathcal{C} \otimes$ Vec is isomorphic to the free module functor $F_{A}: \mathcal{C} \rightarrow \mathcal{C}_{A}, F_{A}(X)=$ $X \otimes A$. See [曰, Corollary 3.30 and Remark 3.31 (i)]. It follows from this that $\mathcal{C}_{A}^{0}$ is a non-degenerate braided fusion category and $\mathrm{FP} \operatorname{dim} \mathcal{C}_{A}^{0}=$ FPdim $\mathcal{C} /(\operatorname{FPdim} A)^{2}$. Moreover, $\left(\mathcal{C}_{A}^{0}\right)^{\text {rev }} \simeq \mathcal{Z}_{2}\left(\mathcal{C}, \mathcal{Z}\left(\mathcal{C}_{A}\right)\right)$ as braided fusion categories.

Proposition 3.1. Let $\mathcal{C}$ be a non-degenerate braided fusion category. Suppose that $A \in \mathcal{C}$ is a connected étale algebra. Then $\mathcal{C}$ is weakly integral (respectively, integral, weakly group-theoretical, solvable or group-theoretical) if and only if $\mathcal{C}_{A}$ is weakly integral (respectively, integral, weakly grouptheoretical, solvable or group-theoretical).

Proof. Observe that the free module functor $F: \mathcal{C} \rightarrow \mathcal{C}_{A}$ is a dominant tensor functor. This implies the 'only if' direction. Suppose now that $\mathcal{C}_{A}$ is in one of the prescribed classes, that is, it is weakly integral, integral, weakly group-theoretical, solvable or group-theoretical. Then the center of $\mathcal{C}_{A}$ is in the same class and, because by $(\boldsymbol{B} \cdot \mathbf{D}), \mathcal{C}$ is equivalent to a fusion subcategory of $\mathcal{Z}\left(\mathcal{C}_{A}\right)$, then $\mathcal{C}$ is in that class as well.

For a non-degenerate fusion category $\mathcal{C}$, we shall denote by $[\mathcal{C}]$ its equivalence class in the Witt group. Recall from [甘, Corollary 5.9] that two non-degenerate braided fusion categories $\mathcal{C}_{1}$ and $\mathcal{C}_{2}$ are Witt-equivalent if and only if there exists a fusion category $\mathcal{D}$ such that $\mathcal{Z}(\mathcal{D}) \cong \mathcal{C}_{1} \otimes \mathcal{C}_{2}^{\text {rev }}$ as braided fusion categories.

In view of the equivalence (B.]), if $A \in \mathcal{C}$ is a connected étale algebra, then $[\mathcal{C}]=\left[\mathcal{C}_{A}^{0}\right]$.

\section{Braided fusion CATEGories AND BRAIDED $G$-CROSSED Fusion CATEGORIES}

Let $G$ be a finite group. Recall that a braided $G$-crossed fusion category [ए:3], [24] is a fusion category $\mathcal{A}$ endowed with a $G$-grading $\mathcal{A}=\oplus_{g \in G} \mathcal{A}_{g}$ and an action of $G$ by tensor autoequivalences $\rho: \underline{G} \rightarrow \underline{\text { Aut }}_{\otimes} \mathcal{A}$, such that 
$\rho^{g}\left(\mathcal{A}_{h}\right) \subseteq \mathcal{A}_{g h g^{-1}}$, for all $g, h \in G$, and a $G$-braiding $c: X \otimes Y \rightarrow \rho^{g}(Y) \otimes X$, $g \in G, X \in \mathcal{A}_{g}, Y \in \mathcal{A}$, subject to compatibility conditions.

A Tannakian subcategory $\mathcal{E}$ of a braided fusion category $\mathcal{C}$ gives rise to a connected étale algebra $A$ in $\mathcal{C}$. If $G$ is a finite group such that $\mathcal{E} \cong \operatorname{Rep} G$ as symmetric categories, then $A$ is the algebra of functions on $G$ with the regular action of $G$.

The fusion category $\mathcal{C}_{A}$ is in this case the de-equivariantization $\mathcal{C}_{G}$ of $\mathcal{C}$ with respect to $\operatorname{Rep} G$, and it is a braided $G$-crossed fusion category.

The braided fusion category $\mathcal{C}_{A}^{0}$ is the neutral component of $\mathcal{C}_{G}$ with respect to the associated $G$-grading.

Conversely, let $\mathcal{A}$ be a $G$-crossed braided fusion category. Then the equivariantization $\mathcal{A}^{G}$ under the action of $G$ is a braided fusion category. The canonical embedding $\operatorname{Rep} G \rightarrow \mathcal{A}^{G}$ of fusion categories is fact an embedding of braided fusion categories. Hence $\mathcal{A}^{G}$ contains $\mathcal{E} \cong \operatorname{Rep} G$ as a Tannakian subcategory.

The $G$-braiding on $\mathcal{A}$ restricts to a braiding in the neutral component $\mathcal{A}_{e}$ of the $G$-grading. Furthermore, the group $G$ acts by restriction on $\mathcal{A}_{e}$ and this action is by braided tensor autoequivalences. This makes the equivariantization $\mathcal{A}_{e}^{G}$ into a braided fusion subcategory of $\mathcal{A}^{G}$. This fusion subcategory coincides with the centralizer $\mathcal{Z}_{2}\left(\mathcal{E}, \mathcal{A}^{G}\right)$ of the Tannakian subcategory $\mathcal{E}$ in $\mathcal{A}^{G}$. See [ए]6].

In this way, equivariantization defines a bijective correspondence between equivalence classes of braided fusion categories containing $\operatorname{Rep} G$ as a Tannakian subcategory and $G$-crossed braided fusion categories [ए5], [प6], [प], Section 4.4]. The braided fusion category $\mathcal{A}^{G}$ is non-degenerate if and only if the neutral component $\mathcal{A}_{e}$ is non-degenerate and the $G$-grading of $\mathcal{A}$ is faithful [Q, Proposition 4.6 (ii)].

In particular, if $\mathcal{C}$ is a non-degenerate braided fusion category containing a Tannakian subcategory $\mathcal{E} \cong \operatorname{Rep} G$, then $|G|^{2} \operatorname{divides} \operatorname{FPdim} \mathcal{C}$.

Let $\mathcal{A}$ be a $G$-crossed braided fusion category such that the neutral component $\mathcal{A}_{e}$ is non-degenerate and the $G$-grading of $\mathcal{A}$ is faithful. As a consequence of (B] $)$, we have $\mathcal{A}_{e}^{\text {rev }}=\mathcal{Z}_{2}\left(\mathcal{A}^{G}, \mathcal{Z}(\mathcal{A})\right)$ and there is an equivalence of braided fusion categories

$$
\mathcal{Z}(\mathcal{A}) \simeq \mathcal{A}^{G} \otimes \mathcal{A}_{e}^{\mathrm{rev}} .
$$

In this context we have the following refinement of Proposition $\left[\begin{array}{l}\text { L. } \\ \text { : }\end{array}\right.$

Proposition 4.1. Let $\mathcal{C}$ be a braided fusion category. Suppose that $\mathcal{E} \cong$ $\operatorname{Rep} G \subseteq \mathcal{C}$ is a Tannakian subcategory. Then $\mathcal{C}$ is weakly integral (respectively, integral or weakly group-theoretical) if and only if $\mathcal{C}_{G}^{0}$ is weakly integral (respectively, integral, weakly group-theoretical). In addition, $\mathcal{C}$ is solvable if and only if $\mathcal{C}_{G}^{0}$ is solvable and $G$ is solvable. 
Proof. The statement concerning weakly integral, integral and weakly grouptheoretical fusion categories follows from Proposition [.], since the de-equivariantization $\mathcal{C}_{G}$ is an $H$-extension of $\mathcal{C}_{G}^{0}$, for a (normal) subgroup $H$ of $G$. Suppose that $\mathcal{C}$ is solvable. Then the quotient category $\mathcal{C}_{G}$ and hence its fusion subcategory $\mathcal{C}_{G}^{0}$ are solvable as well. Moreover, since $\mathcal{C} \cong\left(\mathcal{C}_{G}\right)^{G}$ is a $G$-equivariantization, then the category $\operatorname{Rep} G$ is equivalent to a fusion subcategory of $\mathcal{C}$ and it is thus solvable. Hence the group $G$ is solvable. If, on the other hand, $\mathcal{C}_{G}^{0}$ and $G$ are solvable, then $\mathcal{C}_{G}$ is solvable because it is an $H$-extension of $\mathcal{C}_{G}^{0}$, for some subgroup $H \subseteq G$. Hence so is $\mathcal{C} \cong\left(\mathcal{C}_{G}\right)^{G}$.

Note that $\mathcal{C}$ is obtained from $\mathcal{C}_{G}^{0}$ by an $H$-extension, where $H \subseteq G$ is a subgroup of $G$ (the support of $\mathcal{C}_{G}$ ) followed by a $G$-equivariantization. Since the class of group-theoretical fusion categories is not stable under the operation of taking extensions, then the property of being group-theoretical is not inherited in general from $\mathcal{C}_{G}^{0}$.

\section{Solvable Braided fusion CATEgories}

Recall that a fusion category $\mathcal{C}$ is called group-theoretical if $\mathcal{C}$ is Morita equivalent to a pointed fusion category.

If $\mathcal{C}$ is a braided fusion category, it is shown in [ष्8, Theorem 7.2] that $\mathcal{C}$ is group-theoretical if and only if $\mathcal{C}$ contains a Tannakian subcategory $\mathcal{E} \cong$ $\operatorname{Rep} G$ such that the de-equivariantization $\mathcal{C}_{G}$ is a pointed fusion category. This immediately implies the following:

Lemma 5.1. Let $\mathcal{C}$ be a group-theoretical braided fusion category. Then either $\mathcal{C}$ is pointed or it contains a nontrivial Tannakian subcategory.

Proposition 5.2. Let $\mathcal{C}$ be a braided solvable fusion category. Assume in addition that $\mathcal{C}$ is integral. Then either $\mathcal{C}$ is pointed or it contains a nontrivial Tannakian subcategory.

Proof. Since $\mathcal{C}$ is solvable, there exist a group $G$ of prime order and a fusion category $\mathcal{D}$ such that $\mathcal{C}$ is equivalent as a fusion category to a $G$ equivariantization or to a $G$-extension of $\mathcal{D}$. In particular, $\mathcal{D}$ is integral and solvable and $\mathrm{FPdim} \mathcal{D}=\mathrm{FPdim} \mathcal{C} /|G|<\mathrm{FP} \operatorname{dim} \mathcal{C}$.

If $\mathcal{C}$ is a $G$-equivariantization of $\mathcal{D}$, then there is a central exact sequence of tensor functors $\operatorname{Rep} G \rightarrow \mathcal{C} \rightarrow \mathcal{D}$ and $\operatorname{Rep} G$ is a Tannakian subcategory of $\mathcal{C}$. See [U], Example 2.5 and Proposition 2.6].

Suppose next that $\mathcal{C}$ is a $G$-extension of $\mathcal{D}$, then $\mathcal{D}$ is a braided fusion category and we may assume inductively that $\mathcal{D}$ contains a Tannakian subcategory, whence so does $\mathcal{C}$, or $\mathcal{D}$ is pointed. The last possibility implies that $\mathcal{C}$ is nilpotent. By [ $\mathbb{8}$, Theorem 6.10], an integral nilpotent braided fusion category is group-theoretical. Then Lemma 1 implies that $\mathcal{C}$ has one of the required properties.

Theorem 5.3. Let $\mathcal{C}$ be a solvable non-degenerate braided fusion category. Then one of the following holds: 
(i) $\mathcal{C}$ contains a nontrivial Tannakian subcategory, or

(ii) $\mathcal{C} \cong \mathcal{B} \otimes \mathcal{I}_{1} \otimes \cdots \otimes \mathcal{I}_{n}$, as braided fusion categories, where $\mathcal{B}$ is a pointed non-degenerate fusion category and $\mathcal{I}_{1}, \ldots, \mathcal{I}_{n}$ are Ising braided categories.

Proof. The proof is by induction on $\mathrm{FPdim} \mathcal{C}$ (note that, since $\mathcal{C}$ is solvable, FPdim $\mathcal{C}$ is a natural integer). In view of Proposition 5.2, we may assume that $\mathcal{C}$ is not integral. We may further assume that $\mathcal{C}$ is prime, that is, $\mathcal{C}$ contains no proper non-degenerate fusion subcategories other than Vec; otherwise, if $\mathcal{D} \subseteq \mathcal{C}$ is a proper non-degenerate fusion subcategory, then $\mathcal{C} \cong \mathcal{D} \otimes \mathcal{Z}_{2}(\mathcal{D}, \mathcal{C})\left[\square\right.$, Theorem 3.13], and both $\mathcal{D}$ and $\mathcal{Z}_{2}(\mathcal{D}, \mathcal{C})$ are solvable non-degenerate. By induction, $\mathcal{D}$ and $\mathcal{Z}_{2}(\mathcal{D}, \mathcal{C})$ satisfy (i) or (ii), and then so does $\mathcal{C}$.

The adjoint subcategory $\mathcal{C}_{a d}$ is a solvable braided fusion category and it is in addition integral, by [山], Proposition 8.27]. If $\mathcal{C}_{a d}=$ Vec, then $\mathcal{C}$ is pointed and we are done. We may assume that $\mathcal{C}_{a d} ¥ \mathrm{Vec}$ and contains no nontrivial Tannakian subcategories (otherwise $\mathcal{C}$ satisfies (i)). By Proposition $5 \mathcal{2}$, we get that $\mathcal{C}_{a d}$ is pointed, and therefore $\mathcal{Z}_{2}\left(\mathcal{C}_{a d}\right) \cong$ sVec. Indeed, $\mathcal{Z}_{2}\left(\mathcal{C}_{a d}\right)$ is pointed and symmetric, therefore it is super-Tannakian, thus $\mathcal{Z}_{2}\left(\mathcal{C}_{a d}\right) \cong \mathrm{sVec}$ in view of the assumption that $\mathcal{C}_{a d}$ contains no Tannakian subcategories.

Hence $\mathcal{C}_{a d}$ is slightly degenerate, and therefore $\mathcal{C}_{a d} \cong \operatorname{sVec} \nabla \mathcal{C}_{0}$, where $\mathcal{C}_{0}$ is a pointed non-degenerate braided fusion category [ $[\mathbb{U}$, Proposition 2.6 (ii)]. But $\mathcal{C}$ is prime, by assumption, and hence $\mathcal{C}_{a d} \cong$ sVec.

Since $\mathcal{C}$ is non-degenerate, then $\mathcal{C}_{a d}=\mathcal{Z}_{2}\left(\mathcal{C}_{p t}, \mathcal{C}\right)$ [ [ $\mathbb{Q}$, Corollary 3.27]. Then we get $\mathcal{C}_{a d}=\mathcal{Z}_{2}\left(\mathcal{C}_{p t}, \mathcal{C}\right) \subseteq \mathcal{C}_{p t}$ and thus $\mathcal{C}_{a d}=\mathcal{Z}_{2}\left(\mathcal{C}_{p t}\right) \cong$ sVec. Appealing again to [W], Proposition 2.6 (ii)], we obtain that $\mathcal{C}_{p t}=\mathcal{C}_{a d}=$ sVec. Then,

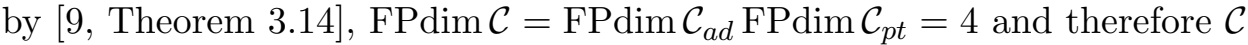
is an Ising braided category. This finishes the proof of the theorem.

\section{The Witt Class of a WEAKLY GROUP-THEORETICAL NON-DEGENERATE BRAIDED FUSION CATEGORY}

Let $\mathcal{W}$ be the group of Witt classes of non-degenerate braided fusion categories and let $\mathcal{W}_{p t}$ and $\mathcal{W}_{\text {Ising }}$ be the subgroups of Witt classes of pointed non-degenerate fusion categories and Ising braided categories, respectively.

If $\mathcal{C}$ is a non-degenerate braided fusion category, $\mathcal{C}$ is called completely anisotropic if the only connected étale algebra in $\mathcal{C}$ is $A=\mathbf{1}$. By [四, Theorem 5.13], every non-degenerate braided fusion category is Witt equivalent to a unique completely anisotropic non-degenerate fusion category.

Lemma 6.1. Let $\mathcal{C}$ be a weakly group-theoretical non-degenerate braided fusion category. Suppose that $\mathcal{C}$ is completely anisotropic. Then $\mathcal{C}$ is nilpotent.

Note that, since every braided nilpotent fusion category is solvable [U], Proposition 4.5 (iii)], it follows that $\mathcal{C}$ is also solvable.

Proof. By [G, Corollary 3.8], equivalence classes of indecomposable module categories over $\mathcal{C}$ are parameterized by isomorphism classes of triples 
$\left(A_{1}, A_{2}, \phi\right)$, where $A_{1}, A_{2}$ are connected étale algebras in $\mathcal{C}$ and $\phi: \mathcal{C}_{A_{1}}^{0} \rightarrow$ $\left(\mathcal{C}_{A_{2}}^{0}\right)^{\text {rev }}$ is a braided equivalence. Furthermore, invertible module categories correspond to such triples where $A_{1}=A_{2}=\mathbf{1}$ (see Remark 3.9 loc. cit.).

The assumption that $\mathcal{C}$ is weakly group-theoretical means that there exists an indecomposable module category $\mathcal{M}$ such that $\mathcal{C}_{\mathcal{M}}^{*}$ is nilpotent. Since $\mathcal{C}$ is braided, $\mathcal{M}$ is naturally a $\mathcal{C}$-bimodule category [ [G, Section 2.8]. Consider the $\alpha$-induction tensor functors [ $[\mathbb{Z}]$, Section 5.1]

$$
\alpha^{ \pm}: \mathcal{C} \rightarrow \mathcal{C}_{\mathcal{M}}^{*}
$$

defined by $\alpha^{ \pm}(X)(M)=X \otimes M, X \in \mathcal{C}, M \in \mathcal{M}$. Letting $a_{X, Y, M}$ : $X \otimes(Y \otimes M) \rightarrow(X \otimes Y) \otimes M, X, Y \in \mathcal{C}, M \in \mathcal{M}$, denote the associativity isomorphisms for the $\mathcal{C}$-action on $\mathcal{M}$, the module functor structures on $\alpha^{ \pm}(X), X \in \mathcal{C}$, are given, respectively, by

$$
a_{Y, X, M}^{-1}\left(c_{X, Y} \otimes \mathrm{id}\right) a_{X, Y, M}: \alpha^{+}(X)(Y \otimes M) \rightarrow Y \otimes \alpha^{+}(X)(M),
$$

and

$$
a_{Y, X, M}^{-1}\left(c_{X, Y}^{\mathrm{rev}} \otimes \mathrm{id}\right) a_{X, Y, M}: \alpha^{-}(X)(Y \otimes M) \rightarrow Y \otimes \alpha^{-}(X)(M),
$$

$Y \in \mathcal{C}, M \in \mathcal{M}$.

The assumption that $\mathcal{C}$ is completely anisotropic implies that the module category $\mathcal{M}$ is an invertible $\mathcal{C}$-bimodule category. Therefore the functors $\alpha^{ \pm}$are equivalences of fusion categories [एँ, Proposition 4.2]. Hence $\mathcal{C}$ is nilpotent, as claimed.

Theorem 6.2. Let $\mathcal{C}$ be a non-degenerate braided fusion category. Suppose that $\mathcal{C}$ is weakly group-theoretical. Then $[\mathcal{C}] \in\left\langle\mathcal{W}_{p t}, \mathcal{W}_{\text {Ising }}\right\rangle$. If in addition $\mathcal{C}$ is integral, then $[\mathcal{C}] \in \mathcal{W}_{p t}$.

Proof. The proof is by induction on $\operatorname{FPdim} \mathcal{C}$. We may assume that $\mathcal{C}$ is prime. If $A \in \mathcal{C}$ is a connected étale algebra, then $\mathcal{C}$ is Witt equivalent to the non-degenerate fusion category $\mathcal{C}_{A}^{0}$. Moreover, $\mathcal{C}_{A}^{0}$ is also weakly group-theoretical, since it is a fusion subcategory of a quotient category of $\mathcal{C}$, and $\mathrm{FPdim} \mathcal{C}_{A}^{0}=\mathrm{FP} \operatorname{dim} \mathcal{C} /(\mathrm{FPdim} A)^{2}$. Hence we may assume that $\mathcal{C}$ is completely anisotropic, otherwise the statement follows by induction. By Lemma $\mathbb{L}$, we get that $\mathcal{C}$ is solvable. In particular, being completely anisotropic, $\mathcal{C}$ contains no nontrivial Tannakian subcategory and it follows from Theorem 5.3 that $\mathcal{C} \cong \mathcal{B} \otimes \mathcal{I}_{1} \otimes \cdots \otimes \mathcal{I}_{n}$, as braided fusion categories, where $\mathcal{B}$ is a pointed non-degenerate fusion category and $\mathcal{I}_{1}, \ldots, \mathcal{I}_{n}$ are Ising braided categories. Hence $[\mathcal{C}] \in\left\langle\mathcal{W}_{\text {pt }}, \mathcal{W}_{\text {Ising }}\right\rangle$. Moreover, if $\mathcal{C}$ is integral, then so is $\mathcal{C}_{A}^{0}$, hence we may also assume inductively that $\mathcal{C}$ is completely anisotropic. Then Theorem 5.3 implies that $\mathcal{C}$ is indeed pointed in this case. This finishes the proof of the theorem.

Let $\widetilde{\mathcal{W}}$ denote the subgroup generated by Witt equivalence classes of the fusion categories $\mathcal{C}(\mathfrak{g}, l)$ of integrable highest weight modules of level $l$ over the affinization of a simple finite-dimensional Lie algebra $\mathfrak{g}$. 
By [曰, Remark 6.5], $\mathcal{W}_{p t} \subseteq \widetilde{\mathcal{W}}$. On the other hand, for any Ising braided category $\mathcal{I}$, we have $[\mathcal{I}]=[\mathcal{C}(\mathfrak{s l}(2), 2)]^{m}$, for a unique odd number $m, 1 \leq$ $m \leq 15$ [⿴囗十, Section 6.4 (3)]. Thus the subgroup generated by $\mathcal{W}_{p t}$ and $\mathcal{W}_{\text {Ising }}$ is contained in $\widetilde{\mathcal{W}}$. As a consequence of Theorem $[\mathbf{L} .2$, we get:

Corollary 6.3. Let $\mathcal{C}$ be a non-degenerate braided fusion category. Suppose that $\mathcal{C}$ is weakly group-theoretical. Then $[\mathcal{C}] \in \widetilde{\mathcal{W}}$.

Remark 6.4. Let $s \mathcal{W}$ denote the Witt group of slightly degenerate braided fusion categories introduced in [5]. Recall from loc. cit. that there is a group homomorphism $S: \mathcal{W} \rightarrow s \mathcal{W}$, defined by $S([\mathcal{C}])=[\mathcal{C} \otimes$ sVec $]$, whose kernel is the subgroup of $\mathcal{W}$ generated by the Witt classes of Ising braided categories. It follows from Theorem 6.2 that for every weakly group-theoretical nondegenerate braided fusion category $\mathcal{C}$, we have $S([\mathcal{C}]) \in s \mathcal{W}_{p t}$.

We also point out the following consequence of Theorem [.2:

Corollary 6.5. Let $\mathcal{C}$ be an integral non-degenerate braided fusion category. Suppose $\mathcal{C}$ is weakly group-theoretical (respectively, solvable). Then there exist an integral nilpotent (respectively, cyclically nilpotent) fusion category $\mathcal{D}$ and a pointed non-degenerate completely anisotropic fusion category $\mathcal{B}$ such that $\mathcal{Z}(\mathcal{D}) \cong \mathcal{C} \otimes \mathcal{B}$ as braided fusion categories.

Proof. By Theorem 6.2 , there exist fusion category $\mathcal{D}$ and a pointed nondegenerate fusion category $\mathcal{B}$ such that $\mathcal{Z}(\mathcal{D}) \cong \mathcal{C} \otimes \mathcal{B}$ as braided fusion categories. Moreover, since every Witt class has a unique representative which is completely anisotropic, we may assume that $\mathcal{B}$ is completely anisotropic.

This implies that $\mathcal{Z}(\mathcal{D})$ is integral and weakly group-theoretical, and therefore so is $\mathcal{D}$. Hence there exists an indecomposable module category $\mathcal{M}$ such that $\mathcal{D}_{\mathcal{M}}^{*}$ is nilpotent. Furthermore, if $\mathcal{C}$ is solvable, then so is $\mathcal{Z}(\mathcal{D})$, and therefore there exists $\mathcal{M}$ such that $\mathcal{D}_{\mathcal{M}}^{*}$ is cyclically nilpotent. This implies the corollary, since $\mathcal{Z}(\mathcal{D}) \cong \mathcal{Z}\left(\mathcal{D}_{\mathcal{M}}^{*}\right)$ as braided tensor categories.

\section{SUFFICIENT CONDITIONS FOR A NON-DEGENERATE BRAIDED FUSION CATEGORY TO BE WEAKLY GROUP-THEORETICAL}

Let $\mathcal{C}$ be a fusion category. Let $\operatorname{Irr}(\mathcal{C})$ be the set of isomorphism classes of simple objects of $\mathcal{C}$ and let $G(\mathcal{C})$ be the group of isomorphism classes of invertible objects.

The group $G(\mathcal{C})$ acts on the set of isomorphism classes of simple objects by tensor multiplication. For a simple object $X \in \mathcal{C}$ let $G[X]$ denote the stabilizer of $X$ under this action. Thus $G[X]$ is a subgroup of $G(\mathcal{C})$ of order dividing $(F P \operatorname{dim} X)^{2}$. Moreover, for every $X \in \operatorname{Irr}(\mathcal{C})$, we have an isomorphism

$$
X \otimes X^{*} \cong \bigoplus_{g \in G[X]} g \oplus \bigoplus_{\substack{Y \in \operatorname{Irr}(\mathcal{C}) \\ \text { FPdim } Y>1}} \operatorname{Hom}_{\mathcal{C}}\left(Y, X \otimes X^{*}\right) \otimes Y .
$$


Lemma 7.1. Let $\mathcal{C}$ be a braided fusion category. Suppose that $\mathcal{C}$ contains no nontrivial non-degenerate or Tannakian fusion subcategories. Then $\mathcal{C}$ is slightly degenerate and the following hold:

(i) $\mathcal{C}_{p t}=\mathcal{Z}_{2}(\mathcal{C}) \cong \mathrm{sVec}$;

(ii) $G[X]=1$, for all simple object $X \in \mathcal{C}$.

Proof. Consider the Müger center $\mathcal{Z}_{2}(\mathcal{C})$ of $\mathcal{C}$. Then $\mathcal{Z}_{2}(\mathcal{C})$ is a symmetric fusion subcategory and therefore it is super-Tannakian. The assumptions on $\mathcal{C}$ imply that $\mathcal{Z}_{2}(\mathcal{C}) \nsubseteq$ Vec and also that $\mathcal{Z}_{2}(\mathcal{C})$ contains no nontrivial Tannakian subcategories. Hence $\mathcal{Z}_{2}(\mathcal{C}) \cong$ sVec [山], Section 2.4] and therefore $\mathcal{C}$ is slightly degenerate. Note that $\mathcal{Z}_{2}(\mathcal{C}) \subseteq \mathcal{C}_{p t}$. Moreover, since $\mathcal{C}_{p t}$ cannot contain any nontrivial non-degenerate or Tannakian fusion subcategory, then it is slightly degenerate as well. By [प], Proposition 2.6 (ii)], every slightly degenerate pointed braided fusion category factorizes in the form $\mathrm{sVec} \otimes \mathcal{B}$, where $\mathcal{B}$ is a pointed non-degenerate braided fusion category. This implies that in our case $\mathcal{C}_{p t}=\mathcal{Z}_{2}(\mathcal{C}) \cong$ sVec, whence we get part (i). Let $\mathbf{1} \neq g \in \mathrm{sVec}$ be the unique nontrivial (fermionic) invertible object. If $X \in \mathcal{C}$ is a simple object, we have $g \otimes X \not X$ [Ш], Proposition 2.6 (i)]. This implies part (ii), since by (i), $g$ is the only nontrivial invertible object of $\mathcal{C}$.

It is well-known that if all the character degrees of a finite group $G$ are powers of a prime number $p$, then $G$ is solvable [1]]. The following theorem extends this result to braided fusion categories. Some instances of the theorem were obtained previously in [피, Theorem 7.3] and [20], Theorem 1.1].

Theorem 7.2. Let $\mathcal{C}$ be a braided fusion category such that $\mathrm{FPdim} \mathcal{C} \in \mathbb{Z}$. Suppose that $p$ is a prime number such that $\mathrm{FPdim} X$ is a power of $p$, for all simple object $X \in \mathcal{C}$. Then $\mathcal{C}$ is solvable.

Note that since the Frobenius-Perron dimension of $\mathcal{C}$ is an integer, we have $(\text { FPdim } X)^{2} \in \mathbb{Z}$, for all $X \in \operatorname{Irr}(\mathcal{C})$ [四, Proposition 8.27]. Therefore the possible powers of $p$ that can occur as simple Frobenius-Perron dimensions in $\mathcal{C}$ are half-integer powers.

Proof. The proof is by induction on $\mathrm{FPdim} \mathcal{C}$. We may assume that $\mathcal{C}$ is integral. Otherwise, $\mathcal{C}$ is a $U(\mathcal{C})$-extension of its integral fusion subcategory $\mathcal{C}_{a d}$, where $U(\mathcal{C})$ denotes the universal grading group of $\mathcal{C}$. By induction, $\mathcal{C}_{a d}$ is solvable. Since $\mathcal{C}$ is braided, its universal grading group is abelian, and therefore $\mathcal{C}$ is also solvable.

It will be enough to show that $\mathcal{C}$ contains a nontrivial Tannakian subcategory $\mathcal{E}$. In such case, $\mathcal{E} \cong \operatorname{Rep} G$ for some finite group $G$ and $G$ is solvable, because $\operatorname{dim} Y=p^{m}, m \geq 0$, for all simple objects $Y \in \operatorname{Rep} G$. Moreover, it follows from [ [3, Corollary 2.13], that the Frobenius-Perron dimensions of simple objects in the de-equivariantization $\mathcal{C}_{G}$, and thus also in its fusion subcategory $\mathcal{C}_{G}^{0}$, are powers of $p$ as well. Since $\operatorname{FPdim} \mathcal{C}_{G}^{0} \leq \operatorname{FPdim} \mathcal{C}_{G}=$ 
FPdim $\mathcal{C} /|G|<\mathrm{FP} \operatorname{dim} \mathcal{C}$ and $\mathcal{C}_{G}^{0}$ is braided, then $\mathcal{C}_{G}^{0}$ is solvable, by induction. Hence so is $\mathcal{C}$, by Proposition 4.$]$.

In view of the relations $(\mathbb{C} \mathbb{C})$, the assumption implies that for every simple object $X$ of $\mathcal{C}$ the order of the group $G[X]$ is divisible by $p$. If $\mathcal{C}$ contains no nontrivial non-degenerate or Tannakian subcategories, then Lemma $\mathbb{R}$ applies, and we obtain that $G[X]=\mathbf{1}$, for all simple object $X \in \mathcal{C}$, which is a contradiction.

We may thus assume that $\mathcal{C}$ contains a nontrivial non-degenerate fusion subcategory. Suppose first that $\mathcal{C}$ is itself non-degenerate. Since $p$ divides FP $\operatorname{dim} \mathcal{C}_{p t}$, then $\mathcal{C}_{p t} \neq$ Vec. Hence $\mathcal{C}_{a d}=\left(\mathcal{C}_{p t}\right)^{\prime} \subsetneq \mathcal{C}$ and, by induction, $\mathcal{C}_{a d}$ is solvable. Then so is $\mathcal{C}$, because it is a $U(\mathcal{C})$-extension of $\mathcal{C}_{a d}$ and $U(\mathcal{C})$ is abelian. If, on the other hand, $\mathcal{D} \subsetneq \mathcal{C}$ is a nontrivial non-degenerate fusion subcategory, then $\mathcal{C} \cong \mathcal{D} \otimes \mathcal{D}^{\prime}$ and $\mathrm{FP} \operatorname{dim} \mathcal{D}, \mathrm{FP} \operatorname{dim} \mathcal{D}^{\prime}<\mathrm{FPdim} \mathcal{C}$. Hence $\mathcal{D}$ and $\mathcal{D}^{\prime}$ are both solvable by induction and therefore so is $\mathcal{C}$. This completes the proof of the theorem.

Corollary 7.3. Let $\mathcal{C}$ be a non-degenerate braided fusion category and let $p$ be a prime number. Suppose that $\mathrm{FP} \operatorname{dim} \mathcal{C}=p^{a} c$, where $a \geq 0$ is an integer, and $c$ is a square free natural number. Then $\mathcal{C}$ is solvable.

Proof. Let $X \in \mathcal{C}$ be a simple object. Since $\mathcal{C}$ is non-degenerate, then $(\text { FPdim } X)^{2}$ divides FPdim $\mathcal{C}$ [U], Theorem 2.11 (i)]. If $\mathcal{C}$ is integral, then FPdim $X$ must be a power of $p$ for all $X \in \operatorname{Irr}(\mathcal{C})$ and therefore $\mathcal{C}$ is solvable, by Theorem [.2. Suppose next that $\mathcal{C}$ is not integral. Then $\mathcal{C}$ is a $G$ extension of an integral fusion subcategory $\mathcal{D}$, where $G$ is an elementary abelian 2-group [ㅍ. Theorem 3.10]. Again in this case, we get that the Frobenius-Perron dimension of a simple object of $\mathcal{D}$ is a power of $p$ and therefore the braided fusion category $\mathcal{D}$ is solvable, by Theorem $\mathbb{T} 2$. Then $\mathcal{C}$, being a $G$-extension of $\mathcal{D}$, is also solvable.

Theorem 7.4. Let $p$ and $q$ be prime numbers. Let $\mathcal{C}$ be a non-degenerate braided fusion category such that $\mathrm{FP} \operatorname{dim} \mathcal{C}=p^{a} q^{b} c$, where $a$ and $b$ are nonnegative integers, and $c$ is a square-free natural number. Then $\mathcal{C}$ is weakly group-theoretical.

Proof. Observe that, after eventually replacing $c$ by an appropriate divisor, we may assume that $c$ is relatively prime to $p$ and $q$. The proof of the theorem is by induction on $\mathrm{FPdim} \mathcal{C}$. As in the proof of Theorem $\mathbb{Z} \cdot 2$, we may assume that $\mathcal{C}$ is integral and it will be enough to show that $\mathcal{C}$ contains a nontrivial Tannakian subcategory.

Let us assume that $\mathcal{C}$ is not nilpotent (and in particular it is not pointed), otherwise there is nothing to prove. Since $\mathcal{C}$ is non-degenerate, then for every simple object $X \in \mathcal{C}$, we have that $(F P \operatorname{dim} X)^{2}$ divides FPdim $\mathcal{C}$. Hence $a \geq 2$ or $b \geq 2$ and moreover, for every simple object $X$, we have FPdim $X=p^{n} q^{m}$, for some $n, m \geq 0$.

Suppose first that $\mathcal{C}$ has no non-invertible simple object of prime power dimension. Then $p q \mid \mathrm{FPdim} X$, for all non-invertible $X \in \operatorname{Irr}(\mathcal{C})$. In view 
of the relations $(\mathbb{R})$, this implies that for any fusion subcategory $\mathcal{D}$, such that $\mathcal{D}$ is not pointed, the Frobenius-Perron dimension of $\mathcal{D}_{p t}=\mathcal{D} \cap \mathcal{C}_{p t}$ is divisible by $p q$. In particular, $\mathrm{FP} \operatorname{dim} \mathcal{C}_{p t} \cap \mathcal{C}_{a d}$ is divisible by $p q$ and thus it is bigger than 2. But, since $\mathcal{C}$ is non-degenerate, then $\mathcal{C}_{p t}=\mathcal{C}_{a d}^{\prime}$ and therefore the category $\mathcal{C}_{p t} \cap \mathcal{C}_{a d}$ is symmetric. It follows that $\mathcal{C}_{p t} \cap \mathcal{C}_{a d}$ contains a nontrivial Tannakian subcategory and we are done.

Suppose next that $\mathcal{C}$ has a simple object of positive prime power dimension. By [س], Corollary 7.2], $\mathcal{C}$ contains a nontrivial symmetric subcategory $\mathcal{D}$. We may assume that $\mathcal{D}$ contains no nontrivial Tannakian subcategory, and thus $\mathcal{D} \cong$ sVec. Since $\mathcal{D}^{\prime \prime}=\mathcal{D}$, then $\mathcal{D}^{\prime}$ is a slightly degenerate fusion category.

If $\mathcal{D}^{\prime}$ has a simple object of odd prime power dimension, then it contains a nontrivial Tannakian subcategory by [ $\square$, Proposition 7.4], and we are done. If $\mathrm{FP} \operatorname{dim} X$ is divisible by $p q$ for all non-invertible simple object $X \in \mathcal{D}^{\prime}$, then $p q$ divides the order of the group $G[X]$ for all non-invertible $X \in$ $\operatorname{Irr}\left(\mathcal{D}^{\prime}\right)$, by $(\mathbb{Z} \mathbb{Z})$. In view of Lemma $\mathbb{Z} \mathbb{D}$, we may assume that $\mathcal{D}^{\prime}$ contains a nontrivial non-degenerate fusion subcategory $\mathcal{B}$. Then $\mathcal{C} \cong \mathcal{B} \otimes \mathcal{B}^{\prime}$, where $\mathcal{B}$ and $\mathcal{B}^{\prime}$ are both non-degenerate. Then $\operatorname{FPdim} \mathcal{B} F \operatorname{dim} \mathcal{B}^{\prime}=\operatorname{FPdim} \mathcal{C}=$ $p^{a} q^{b} c$ and $\mathrm{FPdim} \mathcal{B}, \mathrm{FPdim} \mathcal{B}^{\prime}<\mathrm{FP} \operatorname{dim} \mathcal{C}$. It follows by induction that $\mathcal{B}$ and $\mathcal{B}^{\prime}$ are both weakly group-theoretical and then so is $\mathcal{C}$.

It remains to consider the case where FPdim $X=2^{m}, m \geq 0$, for every simple object $X$ of $\mathcal{D}^{\prime}$. In this case, Theorem $\square \cdot 2$ implies that $\mathcal{D}^{\prime}$ is solvable. Then it follows from Proposition 5.2, that either $\mathcal{D}^{\prime}$ contains a nontrivial Tannakian subcategory, in which case we are done, or $\mathcal{D}^{\prime}$ is pointed. Suppose that $\mathcal{D}^{\prime}$ is pointed. By [ $\square$, Proposition 2.6 (ii)], $\mathcal{D}^{\prime} \cong \mathrm{sVec} \otimes \mathcal{B}$, where $\mathcal{B}$ is a pointed non-degenerate fusion category. If $\mathcal{B}$ is not trivial then, as before, $\mathcal{C} \cong \mathcal{B} \otimes \mathcal{B}^{\prime}$, where $\mathcal{B}$ and $\mathcal{B}^{\prime}$ are both non-degenerate and $\mathrm{FP} \operatorname{dim} \mathcal{B}, \mathrm{FP} \operatorname{dim} \mathcal{B}^{\prime}<\mathrm{FP} \operatorname{dim} \mathcal{C}$, hence $\mathcal{C}$ is weakly group-theoretical, by induction. If, on the other hand, $\mathcal{B} \cong \mathrm{Vec}$, then FPdim $\mathcal{D}^{\prime}=2$ and therefore $\mathrm{FP} \operatorname{dim} \mathcal{C}=\mathrm{FPdim} \mathcal{D} F P \operatorname{dim} \mathcal{D}^{\prime}=4$. Hence $\mathcal{C}$ is nilpotent and in particular it is weakly group-theoretical as well. This completes the proof of the theorem.

\section{Non-DEgEnERATE BRAIDED FUSION CATEGORIES OF LOW DIMENSION}

As an application of Theorem $[\mathrm{C}$ we prove in this section that nondegenerate fusion categories of small dimension are weakly group-theoretical.

Theorem 8.1. Let $\mathcal{C}$ be a weakly integral non-degenerate fusion category such that $\mathrm{FP} \operatorname{dim} \mathcal{C}<1800$. Then $\mathcal{C}$ is weakly group-theoretical.

Proof. Every natural number $n<1800$ such that $n \neq 900$, factorizes in the form $n=p^{a} q^{b} c$, where $p$ and $q$ are prime numbers, $a, b \geq 0$, and $c$ is a square-free integer. In view of Theorem $\mathbf{R . 4}$ it will be enough to consider the case where $\mathrm{FP} \operatorname{dim} \mathcal{C}=900$.

We may assume that $\mathcal{C}$ is a prime non-degenerate fusion category, that is, $\mathcal{C}$ contains no nontrivial proper non-degenerate fusion subcategory, and in 
addition $\mathcal{C}$ contains no nontrivial Tannakian subcategory. Indeed, if $\mathcal{D} \subseteq \mathcal{C}$ is a nontrivial proper non-degenerate fusion subcategory, then $\mathcal{C} \cong \mathcal{D} \otimes \mathcal{D}^{\prime}$ where $\mathcal{D}$ and $\mathcal{D}^{\prime}$ are non-degenerate fusion subcategories of Frobenius-Perron dimension strictly less than 900, and thus weakly group-theoretical. Then $\mathcal{C}$ is weakly group-theoretical in this case. Similary, if $\mathcal{C}$ contains a nontrivial Tannakian subcategory $\mathcal{E} \cong \operatorname{Rep} G$, where $G$ is a finite group, $|G|>1$, then the de-equivariantization $\mathcal{C}_{G}$ is a $G$-crossed braided fusion category of Frobenius-Perron dimension strictly less than 900, whose neutral component $\mathcal{C}_{G}^{0}$ is non-degenerate and thus weakly group-theoretical. Hence $\mathcal{C}_{G}$ and $\mathcal{C}$ are both weakly group-theoretical as well.

It follows from the proof of [U], Theorem 9.2] that a non-degenerate integral fusion category of Frobenius-Perron dimension $p^{2} q^{2} r^{2}$, where $p<q<r$ are prime numbers, contains a nontrivial Tannakian subcategory. Hence we may assume that $\mathcal{C}$ is not integral.

Therefore $\mathcal{C}$ is an $E$-extension of an integral fusion subcategory $\mathcal{D}$, where $E$ is an elementary abelian 2-group [[1], Theorem 3.10]. Then $|E|=2$ or 4 and $\mathrm{FPdim} \mathcal{D}=\mathrm{FPdim} \mathcal{C} /|E|$. Hence we may assume $\mathrm{FPdim} \mathcal{D}=$ $\mathrm{FP} \operatorname{dim} \mathcal{C} / 2$, because otherwise $\mathcal{D}$ and therefore also $\mathcal{C}$ would be solvable, in view of [प], Theorem 1.6]. We may further assume that $\mathcal{D}$ contains no nontrivial non-degenerate or Tannakian fusion subcategories. It follows from Lemma $\mathbb{R}$ that $\mathcal{D}$ is slightly degenerate, $\mathcal{D}_{p t} \cong$ sVec and $G[X]=\mathbf{1}$, for all simple object $X \in \mathcal{D}$.

In addition, if $X \in \mathcal{D}$ is a simple object, then $(\text { FPdim } X)^{2}$ divides $900=$ $F P \operatorname{dim} \mathcal{C}$. Thus FPdim $X=1,2,3,5,6,10$ or 15 . Since the group of invertible objects of $\mathcal{D}$ is of order 2 and $G[X]=1$, then the number of simple objects of $\mathcal{D}$ of a given Frobenius-Perron dimension must be even. In particular, since FPdim $\mathcal{D}=2(15)^{2}$, then $\mathcal{D}$ cannot have simple objects of Frobenius-Perron dimension 15. Also, by [山], Proposition 7.4], $\mathcal{D}$ has no simple objects of Frobenius-Perron dimension 3 or 5. Thus we conclude that the Frobenius-Perron dimension of every non-invertible simple object $X$ of $\mathcal{D}$ is necessarily even. Decomposing $X \otimes X^{*}$ into a sum of simple objects and using that $G[X]=\mathbf{1}$ we arrive to a contradiction; see ([त). This shows that $\mathcal{C}$ is weakly group-theoretical, as claimed.

The result in Theorem 8.0 can be strengthened in the odd-dimensional case. In fact, we have:

Theorem 8.2. Let $\mathcal{C}$ be a weakly integral non-degenerate fusion category such that $\mathrm{FPdim} \mathcal{C}$ is odd and $\mathrm{FPdim} \mathcal{C}<33075$. Then $\mathcal{C}$ is solvable.

Proof. It will be enough to show that $\mathcal{C}$ is weakly group-theoretical, since any odd-dimensional weakly group-theoretical fusion category is necessarily

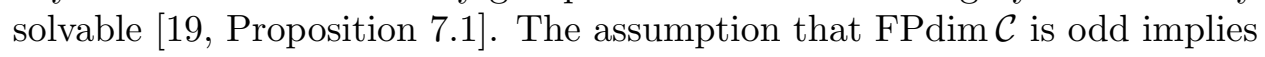
furthermore that $\mathcal{C}$ is integral [ㅍ], Corollary 3.11].

Observe that an odd natural number $n<33075=3^{3} 5^{2} 7^{2}$ such that $n \neq 11025$, factorizes in the form $n=p^{a} q^{b} c$, where $p$ and $q$ are prime numbers, $a, b \geq 0$, and $c$ is a square-free integer. By Theorem $\mathbb{Z}$, we only 
need to consider the case where $\mathrm{FP} \operatorname{dim} \mathcal{C}=11025=3^{2} 5^{2} 7^{2}$. In this case, it follows from the proof of [山], Lemma 9.3] that $\mathcal{C}$ contains a nontrivial symmetric (thus Tannakian) subcategory $\mathcal{E} \cong \operatorname{Rep} G$. Then $\mathcal{C}_{G}^{0}$ is weakly group-theoretical and hence so is $\mathcal{C}$.

\section{REFERENCES}

[1] B. Bakalov and A. Kirillov, Jr., Lectures on tensor categories and modular functors, University Lecture Series 21, Am. Math. Soc., Providence, RI, 2001.

[2] A. Bruguières and S. Natale, Central exact sequences of tensor categories, equivariantization and applications, J. Math. Soc. Jp. 66, 257-287 (2014).

[3] S. Burciu and S. Natale, Fusion rules of equivariantizations of fusion categories, J. Math. Phys. 54013511 DOI: 10.1063/1.4774293 (2013).

[4] A. Davydov, M. Mueger, D. Nikshych and V. Ostrik, The Witt group of nondegenerate braided fusion categories, J. Reine Angew. Math. 677, 135-177 (2013).

[5] A. Davydov, D. Nikshych and V. Ostrik, On the structure of the Witt group of braided fusion categories, Sel. Math., New Ser. 19, 237-269 (2013).

[6] A. Davydov and D. Nikshych, The Picard crossed module of a braided tensor category, Algebra Number Theory 7, 1365-1403 (2013).

[7] P. Deligne, Catégories tensorielles, Mosc. Math. J. 2, 227-248 (2002).

[8] V. Drinfeld, S. Gelaki, D. Nikshych and V. Ostrik, Group-theoretical properties of nilpotent modular categories, preprint arXiv:0704.0195 (2007).

[9] V. Drinfeld, S. Gelaki, D. Nikshych and V. Ostrik, On braided fusion categories I, Sel. Math. New Ser. 16, 1-119 (2010).

[10] P. Etingof, D. Nikshych and V. Ostrik, On fusion categories, Ann. Math. (2) 162, 581-642 (2005)

[11] P. Etingof, D. Nikshych and V. Ostrik, Weakly group-theoretical and solvable fusion categories, Adv. Math. 226, 176-205 (2011).

[12] P. Etingof, D. Nikshych and V. Ostrik, Fusion categories and homotopy theory, Quantum Topol. 1, 209-273 (2010).

[13] S. Gelaki and D. Nikshych, Nilpotent fusion categories, Adv. Math. 217, 10531071 (2008)

[14] M. Isaacs, Character theory of finite groups, Pure and Applied Mathematics 69, Academic Press, New York, 1976.

[15] A. Kirillov, Jr., Modular categories and orbifold models II, preprint arXiv:0110221.

[16] M. Müger, Galois extensions of braided tensor categories and braided crossed G-categories, J. Algebra 277, 256-281 (2004).

[17] M. Müger, Galois theory for braided tensor categories and the modular closure, Adv. Math. 150, 151-201 (2000).

[18] D. Naidu, D. Nikshych and S. Witherspoon, Fusion subcategories of representation categories of twisted quantum doubles of finite groups, Int. Math. Res. Not. 2009 (22) 4183-4219, (2009).

[19] S. Natale and J. Plavnik, On fusion categories with few irreducible degrees, Algebra Number Theory 6, 1171-1197 (2012).

[20] S. Natale and J. Plavnik, Solvability of a class of braided fusion categories, Appl. Categ. Struct. DOI 10.1007/s10485-012-9299-y (2013).

[21] V. Ostrik, Module categories, weak Hopf algebras and modular invariants, Transform. Groups 8, 177-206 (2003).

[22] V. Turaev, Quantum invariants of knots and 3-manifolds, de Gruyter Studies in Math. 18, Berlin, 1994.

[23] V. Turaev, Homotopy field theory in dimension 3 and crossed group-categories, preprint arXiv:math/0005291 (2000). 
[24] V. Turaev, Crossed group-categories, Arabian Journal for Science and Engineering 33, 484-503 (2008).

Facultad de Matemática, Astronomía y Física. Universidad Nacional de Córdoba. CiEM - COniCEt. Ciudad Universitaria. (5000) Córdoba, ArGENTINA

E-mail address: natale@famaf.unc.edu.ar

URL: http://www.famaf.unc.edu.ar/ natale 